\title{
FancD2 Nuclear Foci Absent
}

National Cancer Institute

\section{Source}

National Cancer Institute. FancD2 Nuclear Foci Absent. NCI Thesaurus. Code C84330.

A laboratory test result indicating the absence of Fanconi anemia group D2 protein nuclear foci in cells. 